\title{
BUENOS AIRES: \\ EN BUSCA DE UN MODELO DE GESTIÓN INTEGRAL DEL TRANSPORTE
}

Verónica Mercedes E. Zagare - Buenos Aires

veronica@phicoms.com.ar

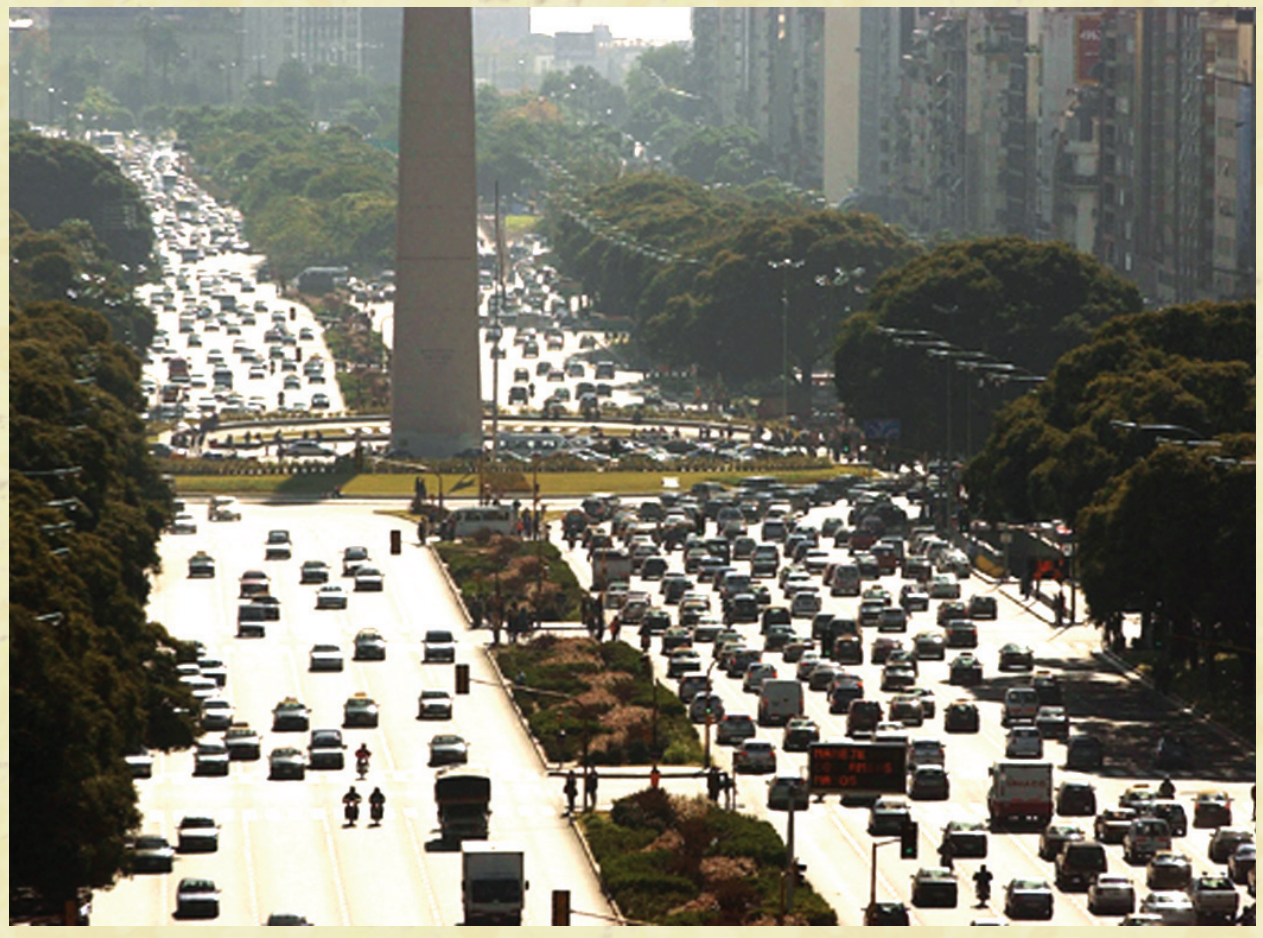

Avenida de Buenos Aires

\section{Resumen}

En la ciudad de Buenos Aires, el modelo de movilidad urbana y sus mutaciones a lo largo de la historia han dejado su impronta en la expansión territorial, y sobre todo, en el proceso de metropolización. En este artículo se analizan esas mutaciones desde varias ópticas. En un primer apartado se estudian algunas de las características relevantes de la ciudad y el Área Metropolitana de Buenos Aires (AMBA) para comprender la relación histórica transporte-territorio. En segundo lugar se hará hincapié en el estudio de la oferta actual de servicios de transporte e infraestructura, según el cambio del modelo de movilidad urbana producido en las últimas décadas. Finalmente, en un tercer apartado se reflexionará acerca de la gestión del transporte en la metrópolis, los actores que intervienen en el proceso y la visión de la problemática en el futuro como un tema clave para resolver. Cabe aclarar que este es un tema central debido a que el AMBA ha sido dirigido históricamente por una pluralidad de gobiernos municipales, conformándose como un escenario fragmentado que impidió una visión integral del territorio en sus continuidades e interacciones. En lo que refiere al transporte, es crucial la implementación de políticas integrales ya que la superposición de competencias es contraproducente para una gestión clara que apunte a un servicio eficiente, sustentable y accesible a todos los grupos sociales.

\section{Palabras clave}

Movilidad urbana - Buenos Aires - Gestión del transporte 
Arquitecto de la Universidad Belgrano Msc. de la Universidad de Torcato Di Bella (Italia)

Especialista en Historia y crítica de la Universidad de Buenos Aires

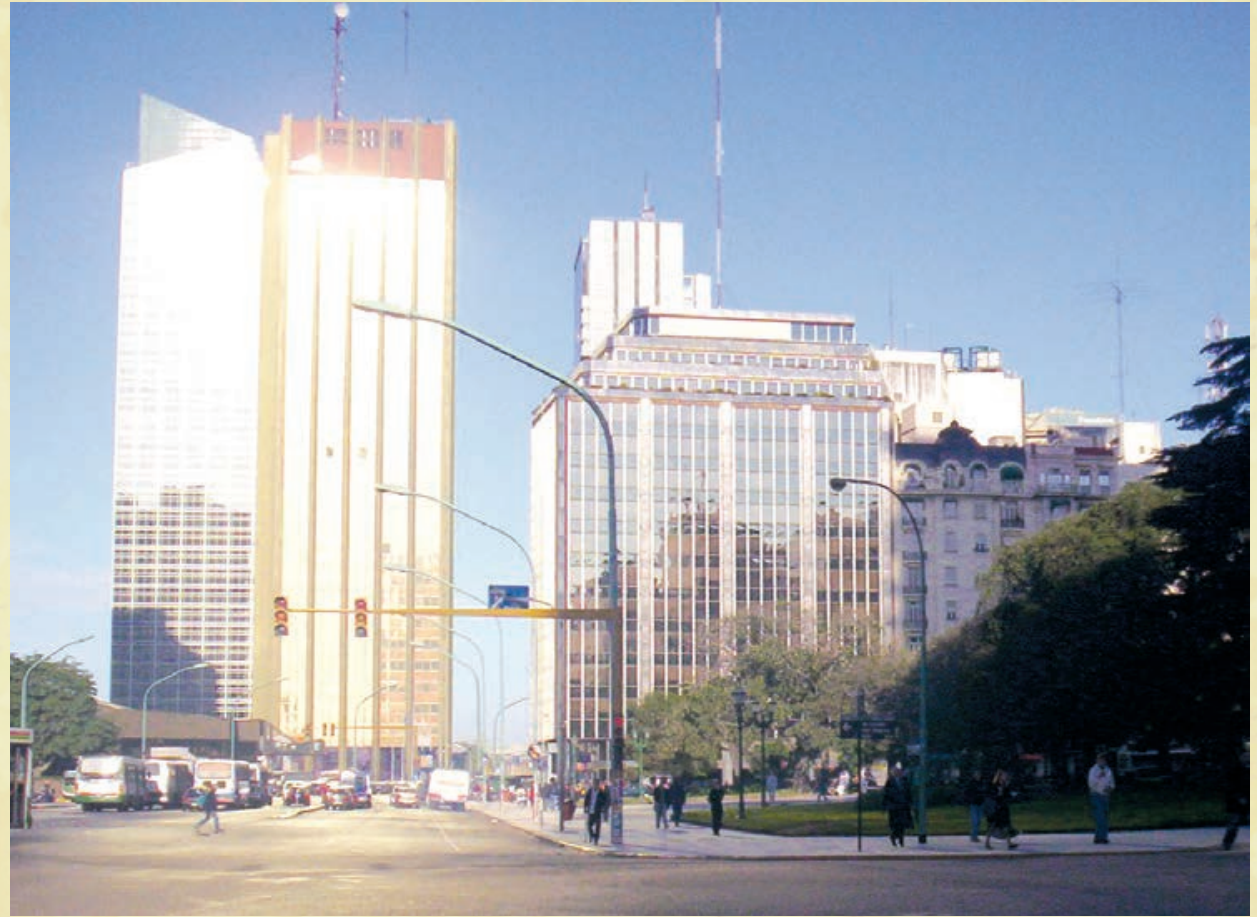

Avenida de Buenos Aires

\section{Summary}

Throughout history, the mutations of the model of urban mobility in Buenos Aires have impacted on the territorial development of the city and its metropolitan region. The aim of this article is to analyze the situation from different perspectives. In the first part, It will be developed a study of the main characteristics of the city and it's Metropolitan Area (AMBA), in order to understand the historic relationship between transportation and territory. In the second part, it will be presented the current transportation and infrastructure offer, according to the changes of mobility patterns during the past two decades. Finally, in a third part, it will be developed an analysis of the management and transportation policies, the actors involved in the process of decision-making and the vision of the future problems in advance.

A coordinated transportation management is a central goal to achieve due to the fragmented political scenery which does not allow thinking the territory as a net of continuities and interactions. It is vital an integral implementation of policies in order to make the public transportation system efficient, sustainable and accessible to all social groups.

\section{Key words}

Urban mobility - Buenos Aires - Transportation management 
I SASSEN, Saskia. 2003. "Los Espectros de la Globalización”. Buenos Aires; Fondo de Cultura Económica. (p.15-2I).

2 VASCONCELLOS, Eduardo. 1996. "Transporte Urbano, Espaço e Eqüidade". San Pablo; FAPESP.

3 Desde 1994, Ciudad Autónoma de Buenos Aires (CABA).

4 Se entiende como AMBA (Área Metropolitana de Buenos Aires) a la Ciudad Autónoma de Buenos Aires (CABA) y a sus municipios lindantes (Partidos del Conurbano Bonaerense). EI INDEC (Instituto Nacional de Estadísticas y Censos) toma como base para la determinación de los límites del AMBA a la CABA más 24 municipios resultantes de las subdivisiones efectuadas en los 19 partidos que originariamente formaron el AMBA.
Las ciudades en la actualidad representan un factor económico muy importante para las economías nacionales, a partir del incremento paulatino de su participación en el Producto Bruto Interno (PBI). En un territorio reducido se concentra por un lado un gran número de población altamente activa, y por otro lado numerosas empresas que aprovechan el factor de aglomeración para incrementar sus beneficios. Estos factores de concentración, que provienen no sólo de la propia industria sino también de la interacción entre las diversas actividades, sirven para aprovechar un mercado común de trabajo, infraestructura y servicios terciarios de apoyo, universidades y desarrollo e investigación tecnológica. Como consecuencia y, sumado el avance del proceso de globalización, la cuestión urbana deja de limitarse a un tema meramente físico de planificación local, y pasa a convertirse en una cuestión estratégica para el desarrollo de las naciones, marcada por la situación social de sus habitantes, su relación en el territorio y su proyección internacional.

Durante los últimos años, los avances tecnológicos han vuelto al transporte cada vez más rápido y económico en costos relativos. Este factor, entre otros, ha producido una "desnacionalización" del espacio urbano (Sassen, 2003)' en pos de las demandas globales de las economías trasnacionales. La ciudad se vuelve clave para la centralización de actividades financieras y de servicios a la producción y necesita establecer redes para el crecimiento local y global. Esta especialización da lugar a procesos de metropolización que incrementan la densidad poblacional tanto de los centros urbanos como de las periferias. El aumento de la población, las nuevas a ctividades económicas y los procesos cambiantes de urbanización han vuelto al transporte una pieza clave para la cohesión del territorio con los procesos de producción y de reproducción social. (Vasconcellos, 1996) ${ }^{2}$

En la ciudad de Buenos Aires (C.A.B.A.) ${ }^{3}$, el modelo de movilidad urbana y sus mutaciones a lo largo de la historia han dejado su impronta en la expansión territorial, y sobre todo, en el proceso de metropolización. En este artículo se analizarán esas mutaciones desde varias ópticas. En un primer apartado se estudiarán algunas de las características relevantes de la ciudad y el Área Metropolitana de Buenos Aires (AMBA ${ }^{4}$ - Ver Mapa I) para comprender la relación histórica transporte-territorio. En segundo lugar se hará hincapié en el estudio de la oferta actual de servicios de transporte e infraestructura, al tener en cuenta el cambio del modelo de movilidad urbana producido en las últimas décadas. Finalmente, en un tercer apartado se reflexionará acerca de la gestión del transporte en la metrópolis, los actores que intervienen en el proceso y la visión de la problemática del futuro como un tema clave para resolver. Cabe aclarar que este es punto central debido a que el AMBA ha sido dirigido históricamente por una pluralidad de gobiernos municipales, conformándose como un escenario fragmentado que impidió una visión integral del territorio en sus continuidades e interacciones. En lo que refiere al transporte, es crucial una correcta integración ya que la superposición de competencias es contraproducente para una gestión clara que apunte a un servicio eficiente, sustentable y accesible a todos los grupos sociales.

\section{Apartado I}

\section{I.I. El desarrollo inicial de la Ciudad de Buenos Aires.}

Desde su fundación en el siglo XVI, la Ciudad de Buenos Aires ha tenido importancia a nivel estratégico como entrada a través de la cuenca del Río de la Plata. Sus límites fueron extendiéndose junto con un incremento poblacional considerable. La ciudad creció como puerto gracias al esquema de desarrollo que marcó el final del siglo XIX: un modelo agroexportador articulado con el Imperio Británico. La concepción de división internacional del trabajo impulsada por las ideas de David Ricardo sostenía que ante una especialización 


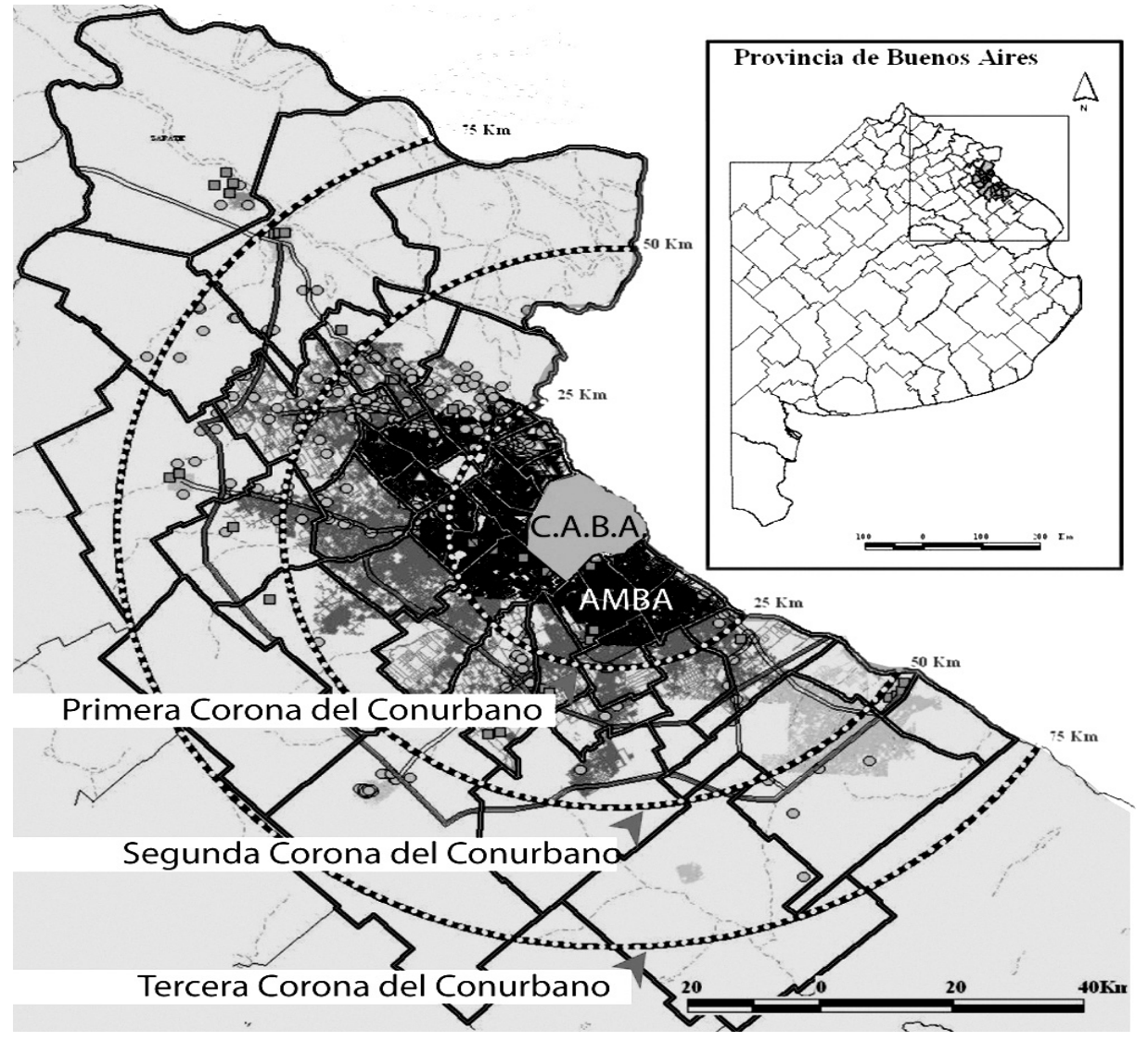

Mapa I. Modelo de movilidad urbana del área metropolitana de Buenos Aires Fuente: Elaboración propia en base a Ciccolella (2002)

racionalmente fundada, el comercio internacional proveería de abastecimiento igualitario para todas las naciones (Ricardo David, 1973)5 ${ }^{5}$. La influencia de estas teorías llevó a Inglaterra a focalizarse en la actividad industrial y Argentina se situó en el mercado internacional como un país exportador de materias primas. (Subsecretaría de Comunicación, Ciudad Autónoma de Buenos Aires, 2002) ${ }^{6}$.

La consecuente demanda de mano de obra sumada tanto al fin de las guerras civiles como a la delicada situación social europea, fueron grandes estímulos para los movimientos migratorios provenientes del viejo continente. Buenos Aires aumentó su población de manera estrepitosa con la llegada de contingentes en su gran mayoría provenientes de Italia, España, Francia, Inglaterra y Alemania ${ }^{7}$. En estos tiempos de exportaciones, el puerto de Buenos Aires se consolidó como la principal vía de salida para las transacciones internacionales.

Hasta mediados del siglo XIX, la población en la ciudad se desplazaba a pie, a caballo, en carruaje particular (los más acaudalados) y en carruaje colectivo a caballo (inspirado en las diligencias de larga distancia). El transporte ferroviario comenzó a funcionar en el país en el año 1857 en Buenos Aires, efectuaba un recorrido de sólo $9 \mathrm{Km}$. que rápidamente se extendió y tuvo una influencia muy marcada sobre el crecimiento físico de la ciudad y la expansión de la mancha urbana. A medida que las redes ferroviarias se ampliaban, se posibilitaba el aprovechamiento de las tierras periféricas y se consolidaba también un sistema de conexión con el resto de las provincias, siendo el tren un motor de crecimiento para la industria nacional (Ver Mapa 2). Alrededor de 1916 se llevó a cabo una electrificación

$5 \quad$ Ricardo, David. 1973. "Principios de Economía Política y Tributación”. México; Fondo de Cultura Económica. (p. 102)

6 Subsecretaría de Comunicación Social, Ciudad Autónoma de Buenos Aires. 2002. "Ciudad de Buenos Aires: de la Fundación a la Participación Ciudadana". Cap.3 "Elementos para la Historia Económica de la Ciudad Autónoma de Buenos Aires". (p.70)

7 La población migrante europea ocupó un porcentaje muy elevado de la población de la Ciudad de Buenos Aires. Entre los años 1869 y 1936 , se calcula que aproximadamente $48 \%$ de la población era italiana, $26.7 \%$ española $7.1 \%$ francesa, $1.6 \%$ inglesa, $1.8 \%$ alemana y $14.8 \%$ argentina. (Fuente, Op. Cit. 6. Cap. I "Memoria y Presente Instirucional de la Ciudad Autónoma de Buenos Aires". p. I7) 
8 Op. Cit. 6. (p.7l). Es necesario dejar en claro que estos crecimientos a lo largo de los dos ejes mencionados todavía tenían lugar dentro de los límites de la ciudad, sin avanzar sobre lo que ahora se considera el territorio conurbano.

9 Este período coincide con una de las etapas de mayor crecimiento industrial. (Op. Cit. 6. -p.72-) paulatina de las líneas que facilitó las conexionesy redujo los tiempos de viaje. El área de influencia de la Ciudad de Buenos Aires se amplió y desarrolló mayormente a lo largo de dos ejes: un eje norte-sur y otro centro-periferia. La localización de la población a lo largo de estos ejes estuvo signada por el nivel socioeconómico (los de mayor poder adquisitivo se instalarían hacia el norte y las distintas generaciones de inmigrantes en la periferia $)^{8}$.

La evolución del trazado férreo creció de los $9 \mathrm{Km}$. iniciales a $2.555 \mathrm{Km}$. de recorrido en menos de veinte años, para luego triplicarse en su periodo de mayor esplendor (I8701914) $)^{9}$ (Ver Cuadro No I).

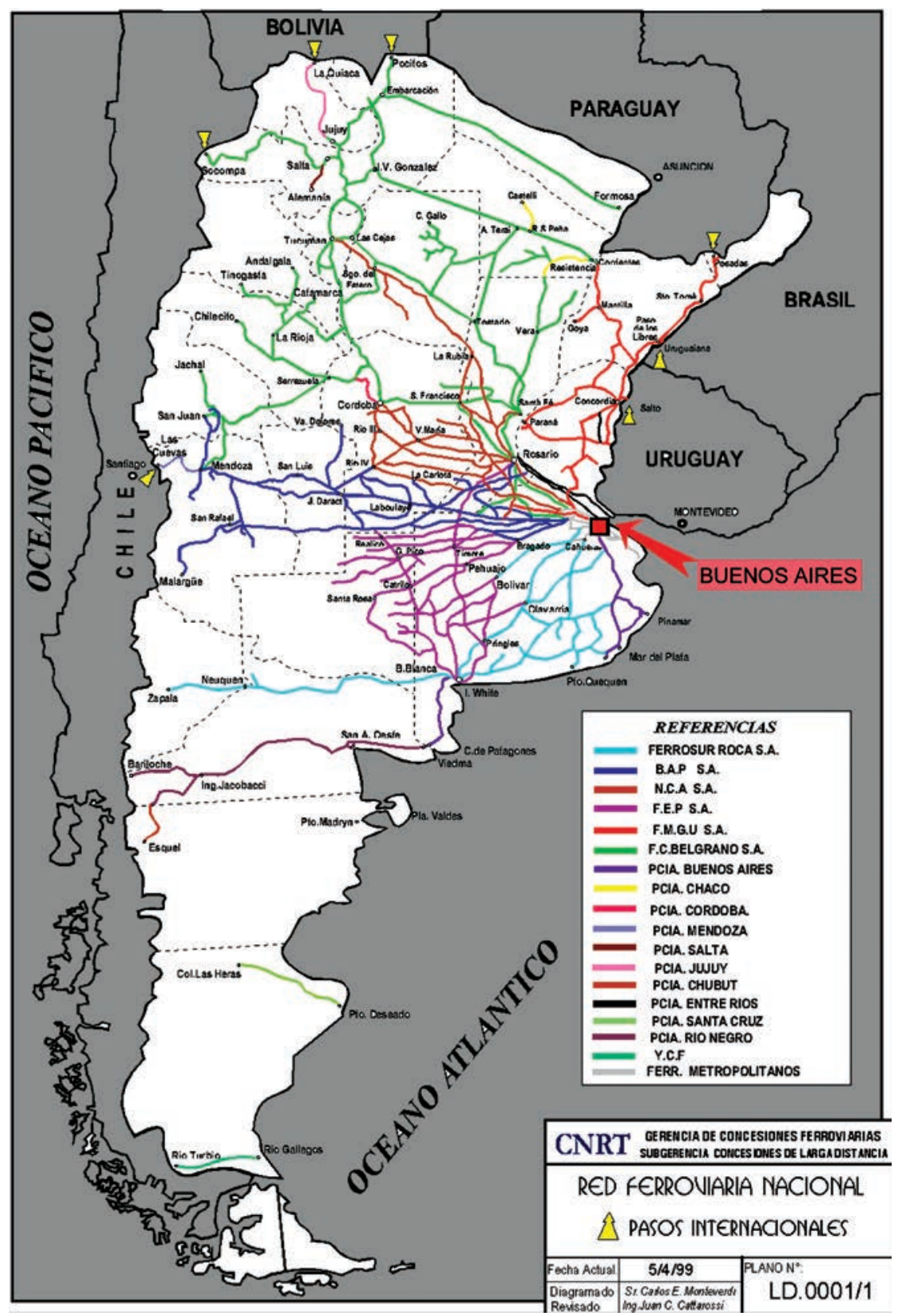

Mapa 2. Red Ferroviaria Nacional 


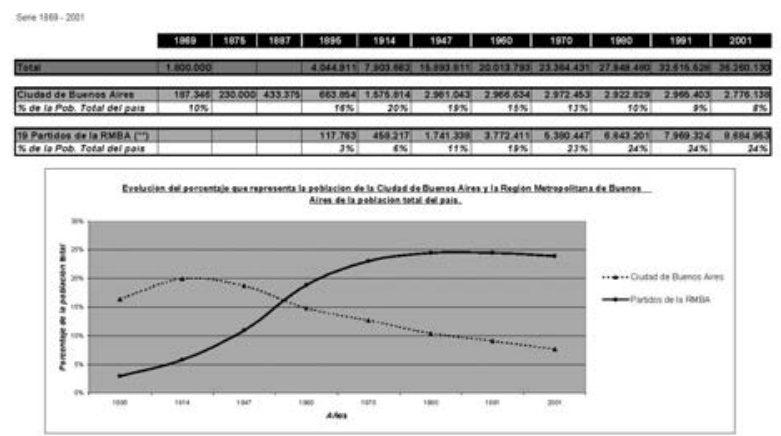

Cuadro I. Evolución de la red ferroviaria

Fuente: Subsecretaría de Comunicación Social de la Ciudad de Buenos Aires (2002) "Ciudad de Buenos Aires, de la fundación a la participación social". Cap. 3: Elementos para la historia económica de la Ciudad de Buenos Aires. Cuadro elaborado por los autores con base a datos del Ministerio de Obras y Servicios Públicos.

Las grandes obras públicas realizadas en este período fueron proyectadas en su mayoría por profesionales franceses, italianos o ingleses, según los cánones de las escuelas de sus países de origen. El resultado: una imagen de ciudad ecléctica con reminiscencias parisinas, grandes avenidas arboladas organizadas a manera de ejes perspectívicos urbanos, imponentes edificios públicos, puertos y estaciones propias de un estilo de arquitectura industrial europea.

Para el año 1898, el transporte de tranvías eléctricos había reemplazado al de tracción sangre al establecer una red que trascendía los límites de la ciudad hasta ese momento construidos, abastecía, pues, a nivel metropolitano. Junto con los autobuses (comenzaron a circular a mediados de los años '20) y a los colectivos (1928) el sistema de conexiones internas se intensificó se logró una cobertura muy amplia. Otro elemento que aparece simultáneamente es el subterráneo, que, siendo el primero en Latinoamérica, expandió su trazado rápidamente hasta mediados de la década del ' 40 , en la que alcanzó un recorrido similar al actual, son modestas las posteriores ampliaciones.

Fue a partir de la década del ' 30 que la crisis del modelo exportador repercutió fuertemente en la economía y la calidad de vida de la sociedad. El servicio de ferrocarriles fue estatizado y sus tarifas reducidas por motivos sociales. La infraestructura existente en la ciudad y las bajas tarifas influyeron en los procesos de suburbanización, guiando a la población hacia terrenos más económicos y alejados del centro (ya que el transporte, por su amplia cobertura y reducidas tarifas dejaba de ser un obstáculo para el desplazamiento). A partir de 1945 y durante casi treinta años, el modelo de Industrialización por Sustitución de Importaciones (ISI) fue el nuevo impulso para el desarrollo económico, que situó al puerto de Buenos Aires como un elemento clave para el corredor portuario Rosario/La Plata, que concentró la mayor actividad productiva del país ya que atrajo otro tipo de migración a la ciudad proveniente del resto de las provincias.

\section{I.2. Buenos Aires en la globalidad}

El modelo neoliberal y la consolidación del Área Metropolitana de Buenos Aires (AMBA)

En la década del '90, luego de la caída del modelo ISI y atravesadas las crisis económicas, políticas y sociales, Argentina se plegó al llamado "capitalismo global” que ya desde los años setenta se vislumbraba, a través de la consolidación de gobiernos de carácter neoliberal. Este modelo se centró en un régimen de apertura económica, desregulación financiera, descentralización y reforma administrativa del Estado. Creció la actividad financiera, por- 
10 PÍREZ, Pedro. 2005. "Descentralización demográfica y Centralización Económica". Revista "Población de Buenos Aires". Año 2, N ${ }^{\circ}$ 2. Editada por la Dirección General de Estadísticas y Censos, Secretaría de Hacienda y Finanzas de la Ciudad Autónoma de Buenos Aires.

II CICCOLELLA, Pablo. 2002. "La Metrópolis Postsocial: Buenos Aires, Ciudad Rehén de la Economía Global” en Actas del Seminario Internacional "El Desafío de las Áreas Metropolitanas en un Mundo Globalizado. Una Mirada a Europa y América Latina", Barcelona, 4,5, y 6 de Junio de 2002, organizado por Institut Catala de Cooperació lberoamericana Institut d'Estudis Territorials - Pontificia Universidad Católica de Chile (p. I)

12 Según datos publicados por CEPAL (Anuario Estadístico de América Latina y el Caribe 2005), Argentina tiene un PBI de I53.014 millones de USS (4.00I USS per cápita), en el cual la ciudad participa con el 53\% (Trivelli, Pablo. 2004. "Realidad y Desafíos de la Ciudad Latinoamericana a Principios del Siglo XXI: Equidad, Competitividad, Sustentabilidad y Gobernabilidad." Presentado en el Curso de Gestión Urbana y Municipa para Centroamérica, organizado por e Instituto de Desarrollo del Banco Mundial. Guatemala.) tuaria y de servicios a la producción, concentrada mayormente en las grandes ciudades. Buenos Aires, la ciudad más poblada del país y sede de gobierno y administración nacional, fue un foco para las inversiones extranjeras directas (IED), arbitrarias y desreguladas. En este marco tuvieron lugar dos procesos: una concentración demográfica en los territorios del Conurbano Bonaerense (Ver Mapa 3), y una concentración económica en el centro de la ciudad ${ }^{10}$. Estos cambios estructurales dieron pie a lo que Pablo Ciccollela denomina "Transición del Proceso de Urbanización" o TPU, proceso que genera nuevas formaciones territoriales "producto de la alteración de las condiciones espaciales y temporales de producción, circulación y consumo" ". Las IED estuvieron principalmente orientadas a las áreas centrales urbanas y a las zonas periféricas del norte del AMBA, establecieron un nuevo mapa del crecimiento metropolitano en el cual las autopistas tomaron el lugar que en algún momento tuvo el trazado ferroviario. Ante una estructura desigual, el impacto de estos capitales extranjeros polarizó aún más el espacio metropolitano, pues quedó conformado en islas. Por un lado, los nuevos emprendimientos residenciales y comerciales de elevado nivel económico, y por otro, a escasos metros, asentamientos ilegales o barrios de medianos o bajos recursos, formados décadas atrás.

En los comienzos, el tren le dio forma a la mancha urbana, y posteriormente las autopistas terminaron por constituir nuevos polos que generaron expansión por sí mismos (Ver Mapa 3), siempre subordinados al núcleo urbano central. Es muy importante analizar estas variaciones en el modelo de movilidad urbana de la ciudad y su región, ya que éste fue el motor generador del espacio físico urbano como lo conocemos en la actualidad, e influyó en la sociedad no sólo como un elemento de conexión sino también como un amplificador de la segregación de algunos sectores. El AMBA concentra el 31\% de la población total del país, y el $35 \%$ de la población urbana en menos del $0.15 \%$ del territorio nacional. Esta relación se hace aún más importante si tenemos en cuenta que la participación de tan bajo porcentaje del territorio en el Producto Bruto Interno (PBI) de la nación es del $53 \% \%^{12}$. La tasa de crecimiento poblacional del AMBA superó con creces la correspondiente a la Ciudad de Buenos Aires, que se mantuvo con población constante durante las últimas décadas (Ver Cuadro 2). El transporte particular automotor no es un medio al que pueda acceder la totalidad de la población, por lo que si éste se convierte en estructurante de los nuevos polos urbanos, evidentemente estaremos frente a un proceso de crecimiento polarizado, en la zona económicamente más productiva del país.

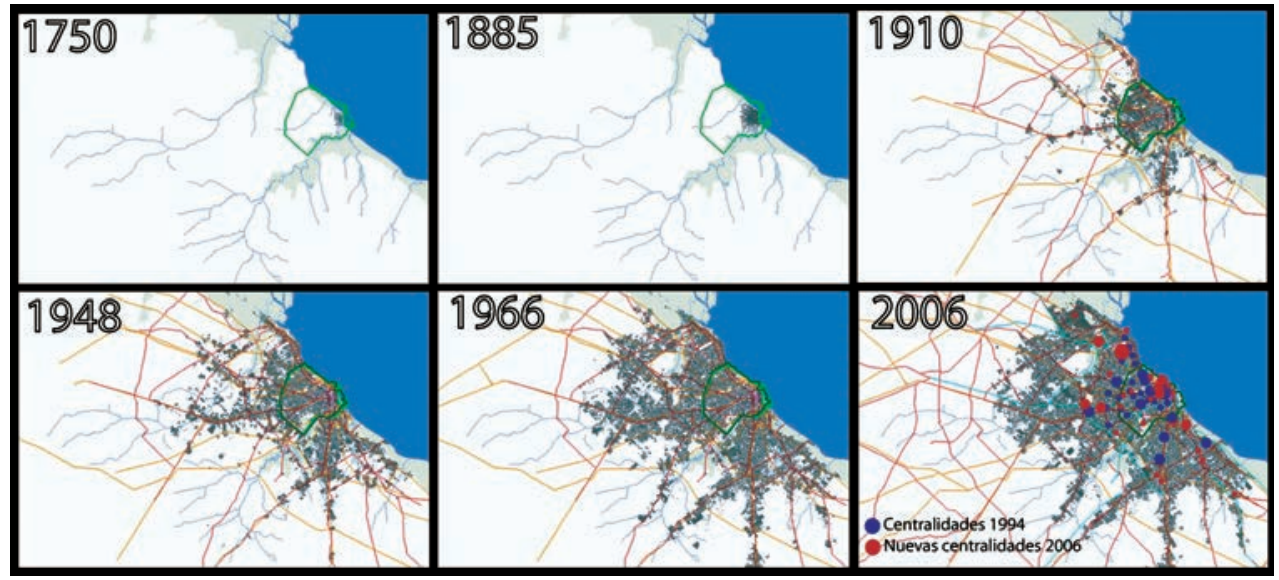

Mapa 3. Evolución de Buenos Aires y su Área Metropolitana Fuente: Elaboracrón con base en los datos del Atlas Ambiental de Buenos Aires (2006) 

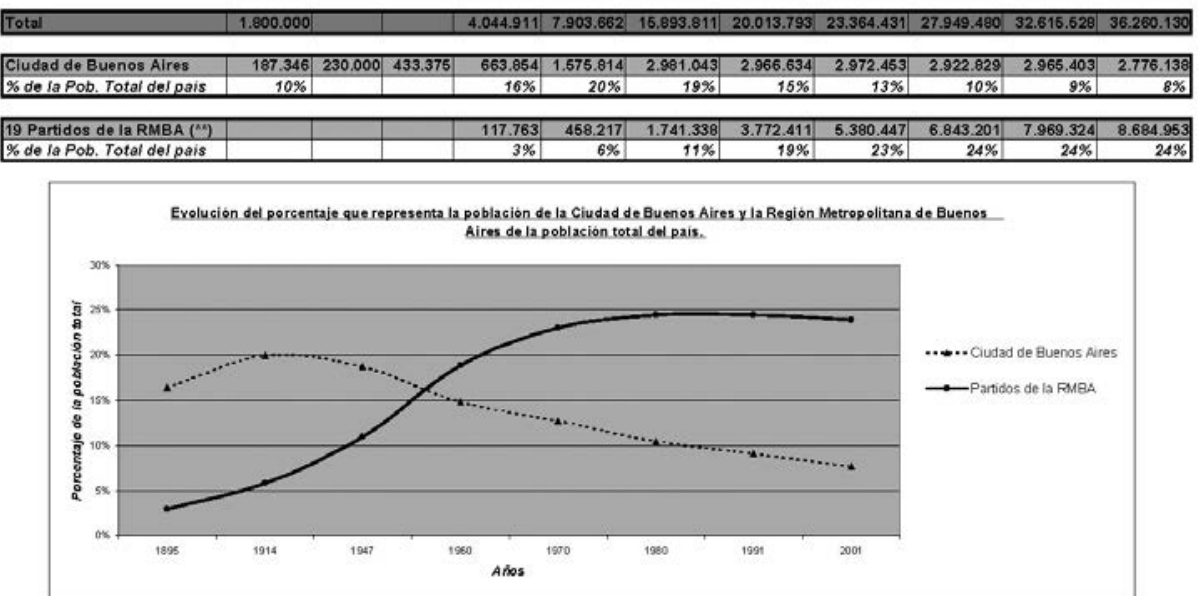

Fuente: Censos Nacionales de Población. Instituto Nacional de Estadísticas y Censos. (INDEC). Serie Histórica

* Algunos componentes de esta juridicción modificaron sus límites por lo cual los datos del 2001 no son

estrictamente comparables con los censos anteriores. Igualmente los datos son válidos a los fines de este análisis.

Cuadro 2. Evolución Poblacional (Total país, ciudad de Buenos Aires y región metropolitana).

\section{Apartado II}

\subsection{Análisis de la oferta de servicios e infraestructura del transporte terrestre en la actualidad en la ciudad y su área metropolitana.}

La ciudad de Buenos Aires y el AMBA poseen una red amplia de transporte terrestre pero la ventaja de modos y cobertura se ve opacada por la complejidad jurisdiccional, que perjudica una gestión integrada del transporte. Si bien la tasa poblacional de la Ciudad de Buenos Aires se mantiene estable desde hace décadas, responde a la población permanente y no a la que diariamente entra a la capital a trabajar, estudiar, o realizar otras actividades. La realidad dista de las cifras oficiales: la ciudad de día no posee dos millones de habitantes, por lo contrario, recibe muchos más, que acceden mediante transporte público o particular. A continuación se expondrá la situación actual desde el punto de vista de la oferta de servicios e infraestructura para dar cuenta de la complejidad y la relevancia que adquiere el manejo del tema en forma coordinada no sólo para reorganizar el territorio a través del sistema sino también para abastecer la fuerte demanda existente.

\section{Ferrocarriles}

Anteriormente hemos ahondado en la importancia de este modo de transporte en la conformación del territorio nacional y sobre todo, de la Ciudad de Buenos Aires, centro del cual partieron desde un comienzo las líneas (Ver Mapa I). A raíz de las privatizaciones de los servicios públicos llevadas a cabo en los años '90, seis empresas son actualmente concesionarias de la red ferroviaria metropolitana, operan siete líneas (Mitre, Sarmiento, Urquiza, Belgrano Norte, Belgrano Sur, Roca y San Martín) y una línea turística diferencial (Tren de la Costa) ${ }^{13}$. Durante este período varios tramos fueron interrumpidos por lo que la red argentina de ferrocarriles que llegó a tener $47.000 \mathrm{~km}$. de largo y ocupar el décimo puesto a nivel mundial redujo a $34.059 \mathrm{~km}$. su extensión. Todas las líneas de ferrocarriles cuentan con subsidios otorgados por el gobierno nacional. 
La cantidad de pasajeros transportados mantuvo una tasa de crecimiento positiva desde sus comienzos hasta el año 2000, en el cual transportó casi 480 millones de pasajeros (Ver Cuadro 3). En el año 200I, y debido a la crisis política, económica, institucional y social por la que atravesó el país, el número de viajes se redujo en casi 100 millones hasta alcanzar los 4 I 2 millones en el año 2005. La estación más importante es Retiro y se encuentra situada al Norte de la ciudad, en las cercanías del puerto. Otra estación importante es Constitución, situada al Sur.

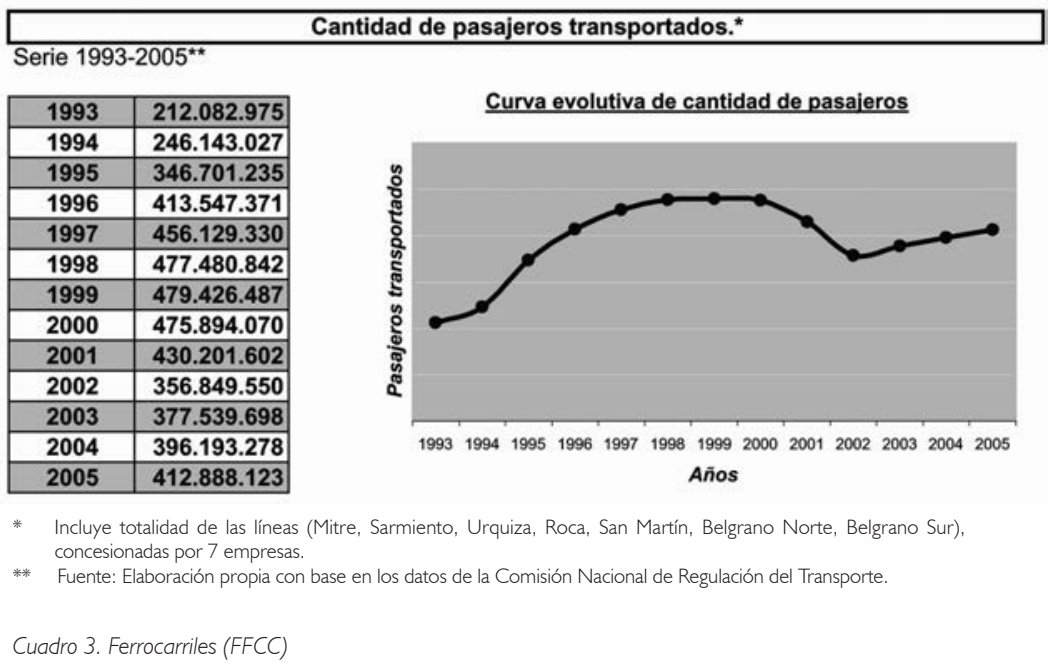

Los transportes de carga están concesionados a otras seis empresas, y han manifestado en general un aumento continuo en las toneladas transportadas desde el año 1993 hasta la actualidad. Si bien todas las líneas sufrieron una disminución importante en las toneladas transportadas durante el período de crisis mencionado (año 200I), pudieron reponerse en los años subsiguientes. La línea que mayor crecimiento ha demostrado es la que une la Ciudad de Buenos Aires con Rosario, Santa Fe, Córdoba y Tucumán, cuya tasa de crecimiento supera ampliamente la media del resto de las líneas. La única línea que no ha podido recuperarse es la correspondiente a Belgrano Cargas (une Buenos Aires con Salta, Tucumán y llega hasta territorio chileno), que actualmente se encuentra en estado crítico en varios tramos.

\section{Subterráneos}

14 Fuente: Comisión Nacional de Regulación del Transporte (CNRT).
Habiendo sido un país pionero en la construcción de redes subterráneas, tuvo un inicio pujante y luego se estancó debido a las crisis mencionadas. En la época de privatizaciones se dio en concesión a la empresa Metrovías S.A. (concesionaria de la línea Urquiza de ferrocarriles). En la actualidad son cinco líneas de subterráneos y una línea del llamado "Premetro" (transita en la superficie). En total llega a los $95 \mathrm{~km}$. de vías, siendo las líneas D y E las más extensas, con $19 \mathrm{~km}$. cada una. Las obras de ampliación de líneas existentes se llevan a cabo desde hace algunos años, pero todavía el subterráneo se mantiene en circulación dentro de los límites de la ciudad y, a pesar de no tener un trazado denso, transporta más de 250 millones de pasajeros al año ${ }^{14}$ (Ver Cuadro 4). A medida que las líneas sigan extendiéndose, aumentará la cantidad de pasajeros $\mathrm{y}$, por ende, se congestionarán más las estaciones centrales. A fin de evitar soluciones que generen más inconvenientes, es necesario revisar el plan de expansión de las líneas de subterráneos. 


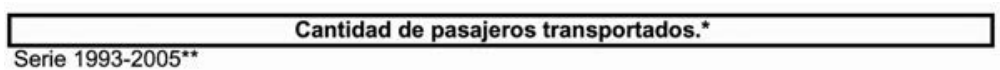

\begin{tabular}{|l|l|}
\hline 1993 & 145.319 .729 \\
\hline 1994 & 171.154 .347 \\
\hline 1995 & 187.220 .075 \\
\hline 1996 & 198.878 .785 \\
\hline 1997 & 221.865 .056 \\
\hline 1998 & 254.006 .700 \\
\hline 1999 & 260.310 .633 \\
\hline 2000 & 258.824 .602 \\
\hline 2001 & 241.743 .735 \\
\hline 2002 & 222.067 .124 \\
\hline 2003 & 228.503 .679 \\
\hline 2004 & 241.186 .229 \\
\hline 2005 & 253.319 .033 \\
\hline
\end{tabular}

* Incluye totalidad de las líneas (A, B, C, D, E y Premetro), todas concesionadas por la empresa concesionadas por 7 empresas.

** Fuente: Elaboración propia con base en los datos de la Comisión Nacional de Regulación del Transporte.

Cuadro 4. Subterráneos (FFCC)

\section{Transporte automotor}

Para completar el análisis del modelo de movilidad de la ciudad no puede dejarse de estudiar el medio de transporte público más utilizado: el transporte automotor en sus distintas formas: colectivo, autobuses (media y larga distancia), taxis, remises, charters y, por último, el automóvil particular.

El colectivo nació en los años `20, frente a la necesidad de transportar más cantidad de personas a un precio menor que los taxis. Así, las líneas de colectivos se articularon con los ferrocarriles, sobre todo en la década del ` 40 , al ser sancionada la ley de alquileres, que congeló el precio de locación y paralizó la movilidad del mercado inmobiliario ${ }^{15}$. La gente, al poder viajar con tarifas reducidas (recordemos que en esa década se había estatizado el ferrocarril y disminuido las tarifas) no veían inconvenientes para localizarse lejos del centro.

En la actualidad existen I 35 líneas manejadas por 100 empresas (a razón de I.4 líneas por empresa), y transportan en promedio 1.500 millones de pasajeros al año ${ }^{16}$. Del total de líneas, sólo 33 se manejan únicamente dentro de los límites de la Ciudad de Buenos Aires, mientras que el resto se maneja a escala metropolitana (Ver Mapa 4).

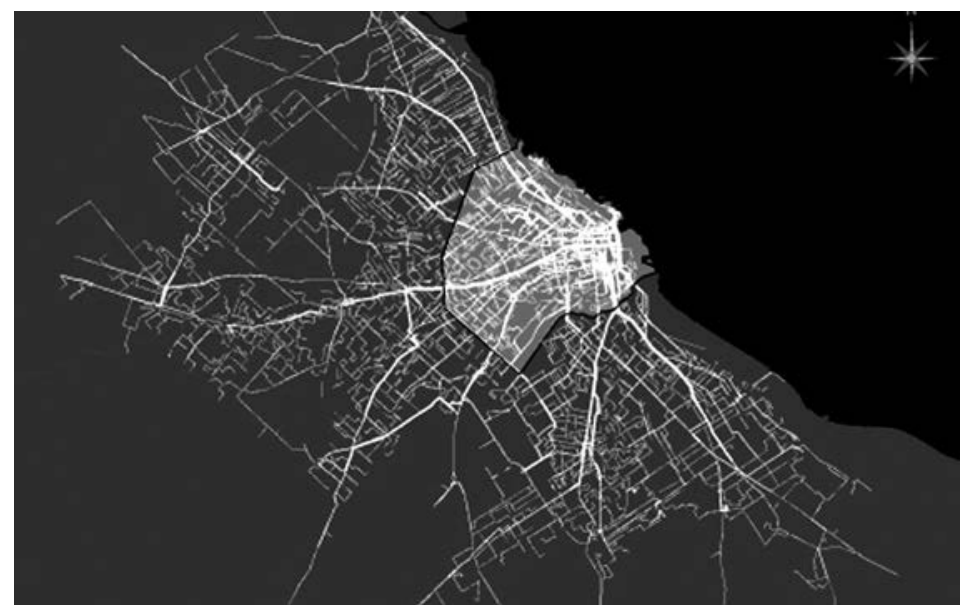

Mapa 4. Densidades de red de colectivos

Fuente: Atlas ambiental de Buenos Aires
15 Op. Cit. 6. (p.75).

16 Fuente: Comisión Nacional de Regulación del Transporte (CNRT). 
17 Fuente: Comisión Nacional de Regulación del Transporte (CNRT)

I8 Fuente: Publicación del Seminario Internacional "Sistemas Integrados de Pasajeros" Organizado por el Ente Único Regulador de Servicios Públicos de la Ciudad de Buenos Aires (EURSP-CABA) y la Facultad de Ciencias Económicas, Universidad de Buenos Aires. 8 de agosto de 2006. Disertación de Julio C. Balbi, Director del EURSP-CABA. (p.4).

19 Op. Cit. 14. (p.4)

20 El circuito se completaría con la construcción de la Autopista Ribereña, la Autopista Illía, la Av. 27 de Septiembre y la ejecución de otros tramos específicos. (Fuente: Gobierno de Buenos Aires: Anillo vial. (http://www.buenosaires.gov.ar).
Otros elementos del sistema de transporte automotor de pasajeros son los autobuses o "micros" de media y larga distancia (empresariales y turísticos). Estos transportes salen de las estaciones más importantes, hacia la provincia o el resto del país. La principal estación es Retiro, que cuenta con 75 plataformas y llega a tener diariamente un servicio de 2000 micros en temporada alta. La concesión de la Terminal de pasajeros Retiro pertenece a la empresa TEBA S.A. Otras estaciones terminales son Liniers (ubicada en el extremo Oeste de la ciudad), y La Plata, a $55 \mathrm{~km}$. del centro, fuera del perímetro de la CABA.

En el año 2005 se calculó un total de 623.407 micros salientes y entrantes a dicha estación. Transportan 57.5 millones de pasajeros anuales (para el año 2005) ${ }^{17}$. Las 135 empresas que manejan este servicio llegaron a tener en 2005 un parque de 3.989 micros en funcionamiento.

En este análisis deben considerarse también los taxis. Actualmente, 88.400 licencias están en vigencia, y circulan un total de 35 mil vehículos ${ }^{18}$. Amén de esta cifra alarmante en cantidad, se tienen que considerar los servicios de charters (vehículos colectivos de capacidad reducida), los remises (similares en funcionamiento a los taxis), además de los automóviles que no estén registrados. Finalmente, y para completar el modelo se debe hacer referencia al transporte automotor particular. Diariamente entran a la ciudad I .6 millones de vehículos particulares $^{19}$, en su mayoría infra-ocupados, que generan, sumados a los autos que circulan dentro de la ciudad, y a los otros modos de transporte ya citados, un caos de tránsito en los puntos centrales urbanos.

\section{Las Autopistas}

La Ciudad de Buenos Aires posee seis conexiones importantes con el área metropolitana: por el Norte (Acceso Norte por General Paz - hacia los partidos de Pilar, Escobar, Zárate, Campana, y demás); por el Oeste (por Autopista Perito Moreno hacia Morón, Moreno y demás.); por el Sudoeste (por Autopista Dellepiane hacia Ezeiza, lugar de ubicación del aeropuerto); por el Sur (hacia Lomas de Zamora y otros); y dos por el Sudeste (uno hacia La Plata -Capital de la provincia de Buenos Aires- por Autopista Buenos Aires-La Plata) y hacia Avellaneda.

Como puede verse en el mapa (Ver Mapa 5), la Ciudad de Buenos Aires está bordeada hacia el Oeste por la Avenida General Paz, que permite recorrer casi todo el perímetro de la ciudad de Norte a Sur. En sus otros bordes (los correspondientes al Sudeste hasta el Noreste, la conexión está interrumpida, no puede ser circunvalada en forma continua. Esta imposibilidad acarrea muchos problemas ya que justamente en los sectores en los que se interrumpe es en donde se necesitaría un tránsito más fluido. A estos fines, en septiembre de 2004 se ha remitido a la Legislatura el proyecto de Anillo Vial, a través del cual se completaría el circuito ${ }^{20}$ y así podría recorrerse la ciudad perimetralmente sin necesidad de entrar. Esto alivianaría mucho el tránsito, especialmente el de carga, y liberaría los accesos al Puerto de Buenos Aires, la Estación Terminal de Ómnibus Retiro y al Aeroparque Jorge Newbery. A su vez se distribuiría más ordenadamente el tránsito y se favorecerían las conexiones hacia los partidos que lindan hacia el Sur, los que siempre fueron más perjudicados en cuanto a lo que a accesos se refiere. Varias de las obras contempladas en el proyecto de Anillo Vial ya han sido ejecutadas, y otras están en proceso. Más allá de las polémicas generadas por la forma de resolución proyectual de algunos de los tramos para ejecutar, la finalización de este anillo beneficiará al tránsito interno, reducirá su densidad y favorecerá al desarrollo económico de partidos que actualmente no se encuentran bien conectados. 


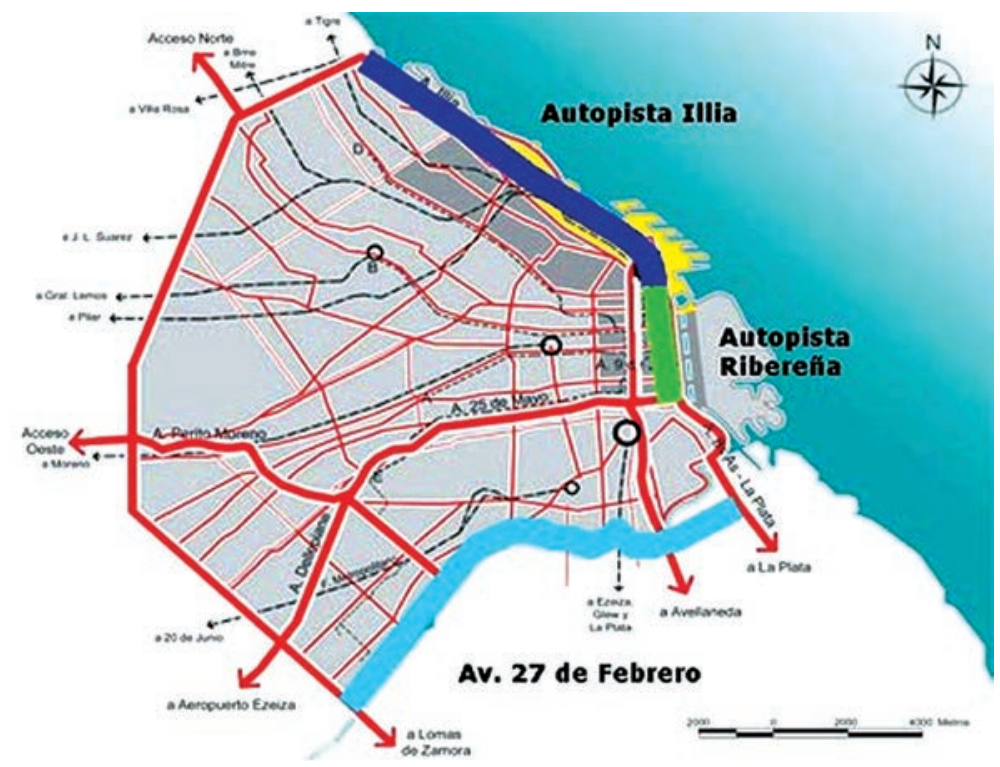

Mapa 5. Sistema Vial de Autopistas

Fuente: http://estatico.buenosaires.gov.ar/areas/obr_publicas/anillo_vial/mapa_anillo_vial.jpg

\section{Apartado 3}

\section{I. En busca del modelo de gestión integral del transporte urbano}

A lo largo de este artículo hemos analizado la relación transporte-desarrollo físico en la Ciudad de Buenos Aires y su Área Metropolitana. El sistema fue determinante para el crecimiento urbano, pero careció de políticas claras que ordenaran el proceso de urbanización. Entre líneas se leen varias cuestiones que es necesario mencionar a la hora de pensar en una gestión integral.

La Ciudad de Buenos Aires oficia de Distrito Federal de la Nación y se encuentra ubicada en la Provincia de Buenos Aires (cuya capital es la ciudad de La Plata). La Ley 19 del I862 establecía que las autoridades nacionales debían residir en el municipio de Buenos Aires, y, 18 años después, otra Ley, la 1029, ordenaba la cesión del territorio de la Ciudad de Buenos Aires a la Nación. De esta forma, la ciudad fue siempre considerada como la Capital Federal, y estuvo gobernada por una autoridad (Intendente), designada por el Poder Ejecutivo. En el año 1994, en pleno desarrollo del modelo neoliberal, se llevó a cabo una reforma administrativa del Estado y se modificó la Constitución Nacional. En este mismo año se declaró la Autonomía de la Ciudad de Buenos Aires ${ }^{21}$ y pasó a tener una Constitución $y$ autoridades elegidas por el pueblo. Frente a estos cambios, se crearon nuevos organismos, y otros siguieron manteniéndose de carácter nacional, ya que todavía la ciudad sigue siendo sede de gobierno, a pesar de que en un momento se presentaron proyectos para su traslado. La situación resultante es una ciudad en la cual confluyen muchas competencias sobre diversos temas en los niveles nacional, provincial y municipal. Ciudad-puerto, sede administrativa y financiera y foco de la mayoría de las inversiones.

Asimismo, nos encontramos con el Área Metropolitana, y hasta incluso con la Región Metropolitana ${ }^{22}$. Todas se interconectan a través de los sistemas de transporte, pero la

21 Pasa a denominarse Ciudad Autónoma de Buenos Aires o CABA.

22 La Región Metropolitana (RMBA) es una caracterización superior al AMBA ya que se agregan los municipios de la tercera corona del conurbano. 
23 PÍREZ, Pedro. 1999. "Gobernabilidad Urbana y Gestión Metropolitana en Buenos Aires: una Cuestión Pendiente". Ponencia a las IV Jornadas Internacionales Estado y Sociedad, Buenos Aires, Noviembre. (p.5). pregunta es quién ejerce competencia en cada caso. Pedro Pírez realizó un estudio exhaustivo acerca de la gobernabilidad en Buenos Aires, y destacó que "es una ciudad sin gobierno en tanto que tiene muchos gobiernos" ${ }^{23}$. Hay temas que exceden la competencia municipal individual y merecen ser tratados de manera integrada, por lo tanto, es de vital importancia una conformación de entidades a nivel metropolitano para así resolver los problemas a una escala mayor. El transporte, como elemento clave para el desarrollo de la ciudad, debe ser pensado a este nivel para fomentar el crecimiento económico y la reproducción social de la población.

\subsection{El manejo de las políticas de transporte en la Ciudad de Buenos Aires}

La estructura de la gestión del transporte es vertical, depende de la Administración Central a través de la Secretaría de Transporte de la Nación, de la cual dependen la Subsecretraría de Transporte y la Comisión Nacional de Regulación de Transporte (CNRT). La primera es la autoridad de aplicación y la última, de control. El órgano regulador en la ciudad es la Subsecretaría de Transporte y en la Provincia es la Dirección Provincial de Transporte (ambas dependientes del Ministerio correspondiente al área). También es necesario contar las estructuras propias de cada municipio, que incrementan el número de actores en los procesos decisorios. Este panorama complejo de autoridades y superposición de

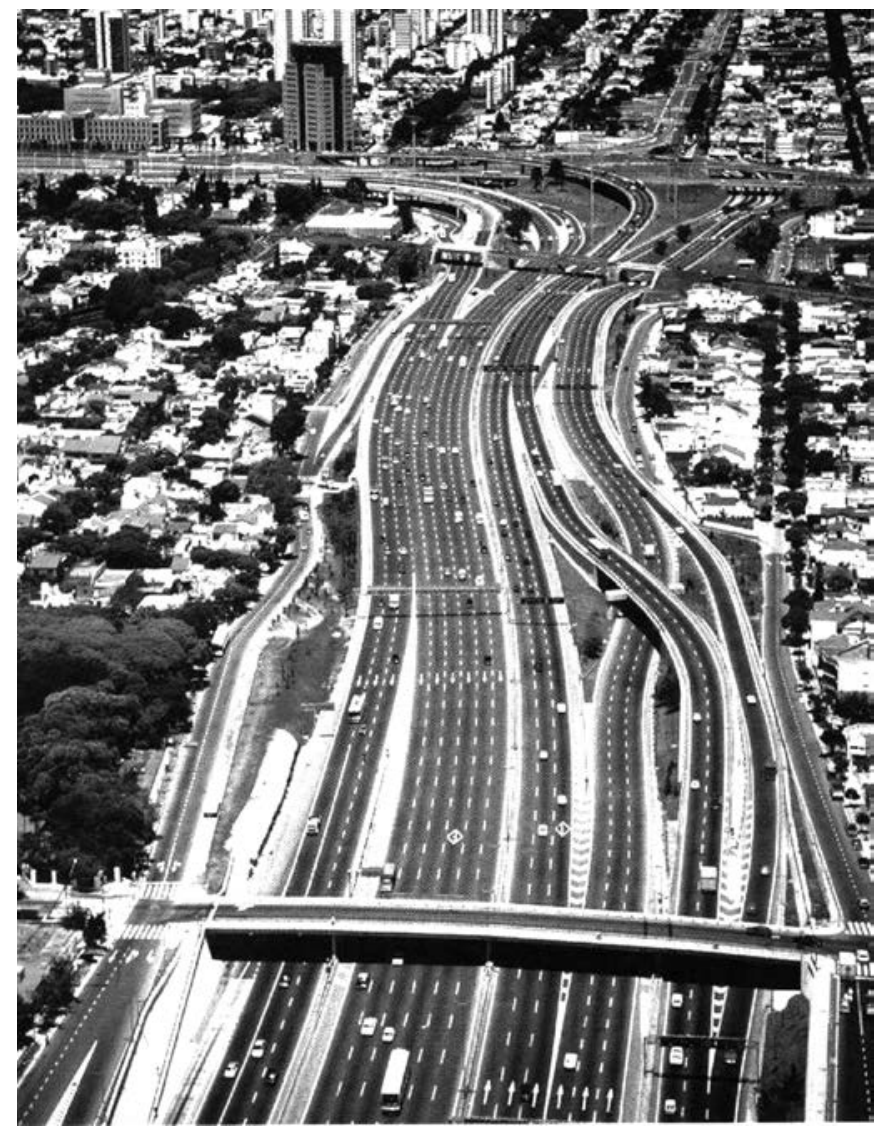

Autopista de Buenos Aires Acceso Norte 
jurisdicciones dificulta la correcta implementación de políticas de gestión que tiendan a un manejo integral del servicio a escala metropolitana. Otros organismos ínter-jurisdiccionales intervienen también en el proceso, como ser el Ente Único Regulador de Servicios Públicos (EURSP-CABA). Todavía no se ha podido establecer un Ente de Coordinación de Transporte a nivel metropolitano, a pesar de haber sido sancionada una ley que así lo establece (Ley 25.03 I), pero es rescatable una iniciativa reciente de la Secretaría de Transporte y la Jefatura de gabinete para crear una agencia coordinadora de transporte a ese nivel.

\subsection{Los problemas que acarrea la falta de coordinación}

En el Seminario Internacional de "Sistemas integrados de pasajeros", un evento organizado por el EURSP-CABA y la Universidad de Buenos Aires, se detectaron las falencias de la desorganización en la gestión de transporte. Los disertantes, representantes de organismos nacionales y provinciales, ${ }^{24}$ coincidieron en general en la necesidad de formulación de políticas de Estado en la materia, a fin de brindar un servicio más eficiente, disminuir los índices de contaminación ambiental e incrementar la funcionalidad del transporte público acorde con el desarrollo económico y la equidad social. La visión compartida se inclina hacia una necesidad de desincentivar el transporte privado, pero esta es una medida que requiere contar con un servicio público adecuado a nivel compensatorio.

Roberto Agosta destaca distintos tipos de falta de integración que pueden encontrarse en el transporte público, así como sus posibles soluciones. La falta de integración institucional podría solucionarse a través de la constitución de una agencia metropolitana que ofrezca una mayor capacidad de control. La falta de integración funcional disminuiría mediante la racionalización de rutas, el establecimiento de carriles exclusivos diferenciados por tipo de transporte, y el establecimiento de una jerarquía que ayude a ordenar las circulaciones por la ciudad. La falta de integración tarifaria debería atacarse por medio de la implementación de tarifas diferenciadas, y así incentivar a los sectores más bajos de la población. Por último, destaca que la falta de integración física puede solucionarse al entender las redes en su conjunto y no por separado, detectar los puntos de transferencia intermodales más importantes para mejorar y aumentar la cantidad de puntos de trasbordo, generar estaciones park-and-ride ${ }^{25}$, y prever el desarrollo del sistema para futuro.

\subsection{Los actores y su participación en el proceso}

El desafío es claro: transportar más pasajeros en menor tiempo, de manera sustentable y accesible a todos los sectores sociales. Quizá en Buenos Aires no se advirtió la problemática en el momento inicial de la expansión, por lo que el crecimiento no pudo ser planificado en torno al desarrollo del sistema de transporte. Es en vano pensar lo que hubiese sido correcto hacer. En muchas ciudades se tomó en cuenta el tema, y se dio el modelo de crecimiento que Robert Cervero ${ }^{26}$ denomina "Adaptive City", en el cual la ciudad se articula desde un comienzo en base a un sistema de transporte previamente planificado. Es vital reconocer que existe actualmente un problema que resolver, y que si no se aúnan esfuerzos desde todos los sectores de la población, es muy difícil revertir la situación. Es posible encontrar una vía de salida para gestar un manejo integral del transporte, que se articule con los aspectos políticos, económicos y sociales. Gracias a los mecanismos de participación impulsados en la última década en la ciudad, se ha podido llegar a discutir con
24 BALBI, Julio César (Director del Ente Único Regulador de los Servicios Públicos de la Ciudad Autónoma de Buenos Aires -EURSPCABA-); TURCO, Nora (Coordinadora de Planificación de Transporte Urbano de la Región Metropolitana de Buenos Aires -Secretaría de Transporte de la Nación-); PÉREZ, Alberto (Vicepresidente de la Comisión de Tránsito y Transporte de la Legislatura de la CABA.); AGOSTA, Roberto (Director del Departamento de Transporte de la Facultad de Ingeniería de la Universidad de Buenos Aires).

25 Las estaciones park-and-ride son sectores hasta los cuales se maneja con automóvil privado, para luego estacionarlo y tomar un servicio público. En algunas ciudades se implementa, pero siempre se realizan estudios de impacto ya que estos centros pueden ser generadores de nuevos polos de congestión vehicular.

26 CERVERO, Robert. 1998. "Transit Metrópolis: A global enquiry”. Islan Press. NY. 
27 http://www.transporte.gov.ar/intrupuba/ intrupuba.html

28 El Plan Urbano Ambiental dibuja la matriz urbano-ambiental a través de la cual se estructurarán las políticas urbanas y ambientales futuras de la Ciudad de Buenos Aires. Su implementación está pautada en la Constitución de la Ciudad de Buenos Aires (Art. 29), y el organismo encargado de formular y actualizar el plan es el Consejo del Plan Urbano Ambienta (creado por la Ley 7I). Lamentablemente no se aprobó todavía por la Legislatura el documento final.

29 http://www.atlasdebuenosaires.gov.ar/ los distintos actores sociales acerca de temas urbanos, donde los técnicos, ciudadanos no especializados y organizaciones sociales (ONG, agrupaciones vecinales y otros) pueden compartir un ámbito de reflexión.

La ciudad ha encarado varias acciones con el fin de analizar la situación actual del modelo de movilidad y plantear las posibles soluciones. Por un lado, la Secretaría de Transporte está llevando a cabo un Plan Integral de Transporte para la Región Metropolitana, y para ellos se realiza una Investigación de Transporte Urbano Público de Buenos Aires (INTRUPUBA) ${ }^{27}$. A su vez, se organizan desde el año 2001 y en torno al Plan Urbano Ambiental ${ }^{28}$, foros de participación sobre la temática, de los cuales se han publicado varios cuadernos de informes técnicos y conclusiones de los talleres, elementos fundamentales para la toma de conciencia ciudadana. Finalmente, otra acción relevante es el incremento de información disponible. Existen en la actualidad datos estadísticos publicados por la CNRT y la Secretaría de Transporte, así como también el Atlas Ambiental de Buenos Aires ${ }^{29}$ (AABA) en el cual se puede acceder a la información histórica, estadística y territorial de las cuestiones estructurales propias de la metrópolis, entre otras, del transporte.

\section{Consideraciones Finales}

Si bien el proceso de metropolización de Buenos Aires estuvo signado por el modelo de movilidad reinante en las distintas épocas, la ausencia de planificación integral de la red de transporte dejó saldos negativos en la conformación espacial, sobre todo en las coronas del Conurbano. En el debate actual sobre este tema, los profesionales en la materia hacen

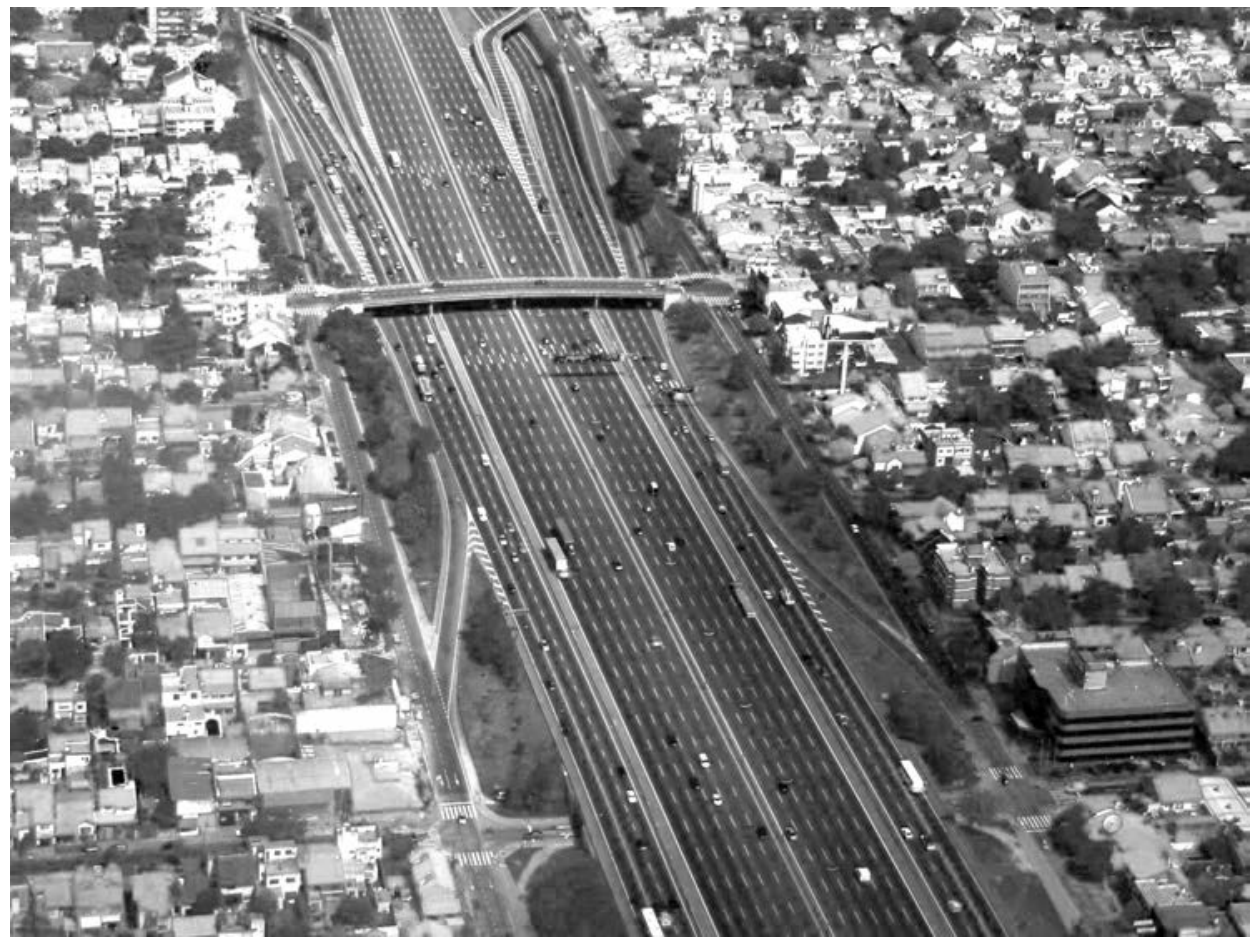

Autopistas de Buenos Aires 
hincapié en la necesidad de una política integral de transporte en la cual el sistema sea entendido como una red multimodal de libre acceso que establezca un mapa de desarrollo territorial y social ordenado, sustentable, y preparado para el crecimiento en el futuro. Felizmente, el debate está instaurado en los ámbitos político y social, por lo que un primer paso está dado. Las acciones referentes al tema son a largo plazo por lo que habrá que esperar por los resultados. La gran ventaja de la discusión de estas cuestiones es que instaura en la ciudadanía la necesidad de pensar el modelo de ciudad en el cual pretenden vivir.

\section{BIBLIOGRAFÍA}

CERVERO, Robert. 1998. “Transit Metrópolis: A global Enquiry”. Islan Press. NY.

CICCOLELLA, Pablo. 2002. "La Metrópolis Postsocial: Buenos Aires, Ciudad - Rehén de la Economía Global" en Actas del Seminario Internacional "El desafío de las áreas metropolitanas en un mundo globalizado. Una Mirada a Europa y América Latina", Barcelona, 4,5, y 6 de Junio de 2002, organizado por Institut Catala de Cooperació Iberoamericana - Institut d'Estudis Territorials - Pontificia Universidad Católica de Chile.

FORMIGA, Nidia. "El Proceso de redistribución espacial en las últimas décadas. El caso del Sudoeste Bonaerense."

PÍREZ, Pedro. 1999. "Gobernabilidad urbana y gestión metropolitana en Buenos Aires: una Cuestión Pendiente". Ponencia en las IV Jornadas Internacionales Estado y Sociedad, Buenos Aires, noviembre.

PÍREZ, Pedro. 2005. "Descentralización demográfica y centralización económica". Revista "Población de Buenos Aires". Año 2, № 2. Editada por la Dirección general de estadísticas y censos, Secretaría de Hacienda y Finanzas de la Ciudad Autónoma de Buenos Aires.

RICARDO, David. 1973. "Principios de economía política y tributación”. México; Fondo de Cultura Económica.

SASSEN, Saskia. 2003. "Los Espectros de la Globalización”. Buenos Aires; Fondo de Cultura Económica.

Subsecretaría de Comunicación Social, Ciudad Autónoma de Buenos Aires. 2002. "Ciudad de Buenos Aires: de la fundación a la participación ciudadana". Cáp.3 "Elementos para la historia económica de la ciudad autónoma de Buenos Aires".

TRIVELLI, Pablo. 2004. "Realidad y Desafíos de la Ciudad Latinoamericana a Principios del Siglo XXI: Equidad, Competitividad, Sustentabilidad y Gobernabilidad.” Presentado en el Curso de Gestión Urbana y Municipal para Centroamérica, organizado por el Instituto de Desarrollo del Banco Mundial. Guatemala.

VASCONCELLOS, Eduardo. 1996. "Transporte Urbano, Espaço e Eqüidade”. San Pablo; FAPESP. 